\title{
Rol de la analgesia y anestesia epidural en cirugía abdominal \\ Francisco Venturelli $M^{1}$, Marcelo Zamorano $D^{1}$, Orlando Felmer $E^{2}$, Marcelo Concha $\mathrm{I}^{3}$.
}

\begin{abstract}
RESUMEN
Desde 1980 que la anestesia peridural (AP) se ha propuesto para manejar el dolor postoperatorio, en especial en cirugías abdominales. A pesar de que ésta es percibida por varios autores, como la analgesia ideal para las cirugías abdominales mayores, hay algunos que prefieren la administración de antiinflamatorios no esteroidales o de opiodes por vía periférica, ya sea en bolos, infusión continua o controlados por el paciente (PCA). Si bien esta última provee mejor analgesia y satisfacción que la administración convencional, no ha demostrado mejorar la morbimortalidad quirúrgica, cosa que sí lo ha hecho la AP. Se realiza una revisión de la literatura con el objetivo de analizar los efectos benéficos y adversos de la anestesia peridural en los pacientes sometidos a una cirugía mayor abdominal. Se concluye que el uso de anestesia epidural intraoperatoria y postoperatoria está asociado a una disminución de la incidencia, severidad de las alteraciones fisiológicas perioperatorias y morbilidad postoperatoria. (Palabras claves/Key words: anestesia epidural/epidural anesthesia; bloqueo sensitivo/sensory block; técnicas anestésicas/anesthetics techniques).
\end{abstract}

\section{INTRODUCCIÓN}

Desde 1980 la anestesia peridural (AP) se ha propuesto para el manejo del dolor postoperatorio, en especial en cirugías abdominales mayores (gastrectomías y hepatectomías, entre otras)․․

La técnica consiste en introducir un catéter a nivel del espacio peridural (entre duramadre y ligamento amarillo), se administra un anestésico local y/u opioide produciendo analgesia y bloqueo simpático. Para cirugías abdominales mayores se debe alcanzar un nivel mínimo de T6, dermatoma que corresponde al apéndice xifoides².

Se ha demostrado que la AP en este tipo de cirugías disminuye el dolor postoperatorio y aumenta la satisfacción de los pacientes ${ }^{3}$.
A pesar de que la AP es percibida para la mayoría de los autores, como la analgesia ideal para las cirugías abdominales mayores, hay otros que prefieren la administración de antiinflamatorios no esteroidales (AINES) o de opiodes, por vía periférica, ya sea en bolos, en infusión o controlados por el paciente. Si bien esta última provee de mejor analgesia y satisfacción de los pacientes que la administración convencional, no ha demostrado mejorar la morbimortalidad de la cirugía, cosa que sí lo ha hecho la $\mathrm{AP}^{3}$.

El objetivo de esta revisión es analizar el estado del arte de los efectos benéficos y adversos de la anestesia peridural en los pacientes sometidos a una cirugía mayor abdominal.

\footnotetext{
Residente de Cirugía. Instituto de Cirugía. Facultad de Medicina. Universidad Austral de Chile.

Cirujano. Instituto Nacional de Enfermedades Respiratorias y Cirugía Torácica.

Anestesiólogo. Instituto Nacional de Enfermedades Respiratorias y Cirugía Torácica.

Correspondencia a: Dr. Francisco Venturelli M. icoventurelli@gmail.com
} 


\section{EFECTOS FISIOLÓGICOS NO ANALGÉSICOS DE LA AP}

\section{SISTEMA CARDIOVASCULAR}

La primera causa de muerte, durante el postoperatorio de una cirugía mayor, es la cardíaca. La excesiva activación de T1-T5 a causa del estrés quirúrgico, produce aumento en la demanda de oxígeno miocárdico y vasoconstricción coronaria, además de desencadenar un estado de hipercoagulabilidad ${ }^{3.4}$.

La AP torácica al producir un bloqueo selectivo de la inervación simpática cardíaca (T1T5), produce disminución del efecto adrenérgico, lo que conlleva una reducción de la presión arterial, frecuencia y contractibilidad cardíaca, los cuales son los mayores determinantes de la demanda de oxígeno miocárdico durante la intervención quirúrgica ${ }^{3}$.

En adición a lo mencionado, el bloqueo produce vasodilatación coronaria y sistémica, aumentando el flujo sanguíneo en las regiones isquémicas del miocardio, como también una reducción en la precarga y poscarga ${ }^{5}$.

Para asegurar los máximos beneficios cardíacos, la AP postoperatoria debe permanecer por 48 a 72 hrs, ya que es en este período cuando se produce la mayor morbilidad cardíaca ${ }^{3}$.

En los estudios controlados aleatorios (ECA) de cirugía de alto riesgo, el grupo de pacientes con anestesia general más AP contra anestesia general única, demostraron menos complicaciones cardíacas en el primer grupo. Yeager et al, evidenció mayor incidencia de infarto, insuficiencia cardíaca, arritmias y angina en el segundo grupo ${ }^{6}$.

Beattie WS et al, realizó un estudio de cohorte prospectivo siguiendo los pacientes con Holter hasta 24 hrs posterior a la cirugía, encontrando en el grupo con AP menos episodios de isquemia ( $17.2 \mathrm{v} / \mathrm{s} 50.0 \%, P=0.01)$, y arritmias (20.7 v/s 50.0\%, P < 0.05). Además, evidenció que los pacientes con epidural presentaron una reducción del riesgo relativo a cualquier evento cardíaco 4 veces menor $(P<0.001)^{7}$.

\section{COAGULACIÓN}

Los eventos tromboembólicos en el período postoperatorio han sido asociados al estado de hipercoagulabilidad producido por el acto quirúrgico. Durante la intervención quirúrgica, se produce una reducción en el flujo venoso dado por: la ventilación mecánica (presión positiva), el bloqueo neuromuscular y la activación del sistema simpático. Este último produce una aumento del factor VIII y de Von Willebrand, inhibe la fibrinolísis a través del PAI-1, disminuye la antitrombina III e inicia la agregación plaquetaria ${ }^{3}$.

La analgesia-anestesia epidural al producir bloqueo simpático, disminuye los efectos de éste sobre el sistema de coagulación, logrando una reducción del riesgo de tromboembólic ${ }^{3}$. Además, esta analgesia, al controlar mejor el dolor postoperatorio, conlleva a una movilización precoz del paciente, disminuyendo más aún el riesgo de trombosis.

Un metanálisis que incluyó 18 trabajos que reportaban trombosis, demostró que el bloqueo neuroaxial reducía el riesgo de esta complicación $(0,56 \text { IC: } 0,43 \text { to } 0,72)^{8}$.

Otros estudios han demostrado que la absorción sistémica del anestésico local actúa como anticoagulante bloqueando el tromboxano A2, reduciendo la viscosidad sanguínea, disminuyendo las proteínas, los eritrocitos y la agregación plaquetaria ${ }^{3}$.

\section{PULMONAR}

La morbilidad pulmonar en el postoperatorio es atribuida tanto al anestésico como a las alteraciones de la función pulmonar. El impacto de la peridural sobre la función pulmonar es ambiguo, sin embargo, se han visto beneficios como: disminuir la instrumentalización de la vía aérea, la necesidad de ventilación mecánica, la incidencia de atelectasia e infecciones pulmonares. ${ }^{4,9}$.

Como es sabido en el postoperatorio inmediato la función pulmonar depende de la relajación muscular residual, de la disfunción diafragmática (ésta se mantiene hasta el 50-7o día postoperatorio), del momento de extubación, de la disminución de la complaciencia pulmonar y del dolor ${ }^{4}$. Con el uso de la AP torácica se requiere menos dosis de relajante muscular, por lo que la recuperación de la función respiratoria es más rápida ${ }^{4}$. Por otro lado, la AP torácica por medio del anestésico local, bloquea el arco reflejo del nervio frénico, permitiendo una mejor función diafragmática ${ }^{4}$.

Se ha demostrado que los pacientes que reciben analgesia epidural son extubados antes, requiriendo menos ventilación mecánica, necesitando menos cuidados intensivos, al igual que menor estadía hospitalaria ${ }^{10}$

Werawatganon $\mathrm{T}$ et al, realizó un metaanálisis, donde concluyó que la analgesia vía 
peridural controlaba mejor el dolor, respecto a la analgesia con opiodes controlada por el paciente. $(95 \% \mathrm{Cl} 1.30 \text { to } 2.19)^{12}$.

Con respecto al aumento del tono vagal, se cree que esto produciría un aumento del tono bronquial, pero en pacientes con cirugía abdominal esto aún no ha sido demostrado ${ }^{4}$.

Posterior a una cirugía abdominal el VEF 1 disminuye, el volumen corriente (VC) puede disminuir hasta en un $60 \%$, alcanzando su pico a las 24-48 hrs. Ambos parámetros pueden demorarse desde una semana hasta 14 días en recuperarse ${ }^{4,10}$. Waba et al demostró que la AP torácica mejora estos dos parámetros encontrando que la reducción de la capacidad residual funcional mejoraba de un $21,7 \%$ a un $15,9 \%$ y que la reducción del VC disminuye de un $63 \%$ a un $45 \%{ }^{13}$. Mankikian ${ }^{14}$ encontró resultad os similares.

Los trabajos que no han demostrado una diferencia a favor de la AP en mejorar los resultados pulmonares, tienen por defecto que incluyen pacientes de bajo riesgo, no controlan la analgesia postoperatoria y/o carecen de significación estadística suficiente ${ }^{3}$.

En resumen, la AP con un anestésico local puede mejorar los resultados pulmonares mediante la atenuación de la respuesta fisiológica a la cirugía, controlando el dolor postoperatorio, mejorando la función de la musculatura respiratoria, permitiendo una extubación más temprana y reduciendo el tiempo de hospitalización.

\section{FUNCIÓN GASTROINTESTINAL}

El íleo postoperatorio, aumenta dramáticamente la morbilidad postoperatoria y los días de hospitalización. Actualmente no hay un tratamiento con el que se obtengan buenos resultados ${ }^{15}$, por lo que su manejo aún es tema de debate.

Antiguamente se creía que el íleo postoperatorio era un factor importante para la cicatrización de las anastomosis intestinales, ya que al estar el intestino con baja o nula motilidad, aseguraba una sutura sin tensión. Actualmente se sabe que una temprana motilidad intestinal, aumenta la irrigación de la zona de sutura, lo que posibilita una rápida cicatrización ${ }^{16}$.

El íleo postoperatorio es causado por ${ }^{16,17}$ : dolor, el uso de óxido nitroso, las altas dosis de opiodes y la respuesta simpática.

La epidural puede reducir el íleo postoperatorio:
1. Aumentando la irrigación de la mucosa intestinal mediante una vasodilatación esplácnica ${ }^{16}$.

2. Manteniendo un pH gástrico cercano a los niveles fisiológicos $3,16,18$.

3. Bloqueando la vía simpática inhibitoria (T5L2) y preservando la estimulación parasimpática intestinal, dada por el nervio vago y sacro, quedando el sistema parasimpático esplácnico sin oposición ${ }^{16,19}$.

4. Mediante el bloqueo del arco reflejo simpático del stress quirúrgico y la disminución del dolor.

5. Disminuyendo la dosis de opioides necesaria ${ }^{16}$.

6. Posibilitando la administración de lidocaína produciendo una disminución de la irritación peritoneal16,20.

Para que la analgesia-anestesia epidural logre acortar el íleo debe cumplir ciertos requisitos: ${ }^{18}$

1. Debe ser instalada antes del stress quirúrgico y de la estimulación de las vías aferentes nocioceptivas.

2. El catéter epidural debe incluir los dermatomos de T5 a L2 y la solución que se inyecta, debe incluir un anestésico local ${ }^{16}$.

3. El anestésico local se debe mantener durante el postoperatorio hasta que la función intestinal regrese.

Hay varios estudios aleatorios que al comparar esta técnica anestésica con la anestesia general más opioides sistémicos, reportan mejores resultados para la primera en cuanto a la recuperación de la función intestinal ${ }^{3}$.

Thoren T, et al y Thörn S-E et al, demostraron que la epidural con anestésicos locales, sin opiodes, acortan más el íleo postoperatorio que con ellos ( $p<0,05$ y $p<0,04$ respectivamente), ${ }^{21}$. Debido a que en cirugías intraperitoneales el dolor es manejado mejor mediante la combinación de opiodies y anestésicos locales, se recomienda esta última opción ${ }^{16}$.

Liu spencer 22 randomizó en forma prospectiva pacientes sometidos a cirugía colónica. Los separó en 4 grupos (MB: anestesia general más epidural torácica con morfina y bupivacaina, $M$ : anestesia general más epidural torácica con morfina, B: anestesia general más epidural torácica con bupivacaina, P: anestesia general más IV-PAC con morfina), el midió la resolución del íleo a través de la eliminación de 
gases por ano y encontró que en los grupo $M B$ y B resolvieron el íleo 35 hrs antes que el M y P $(P<0,05)$.

En un meta-análisis, que incluyó 8 estudios que comparaban la epidural con nivel sobre T12 $\mathrm{v} / \mathrm{s}$ anestesia general en cirugías de abdomen, vieron que la duración del ileo postoperatorio, medido a través de la presencia de flatos y deposiciones fue significativamente menor en el grupo de peridural $(P<0,02)^{16,23}$.

Otro estudio, también prospectivo randomizado, en pacientes sometidos a cirugía de colon laparoscópico demostró que el íleo postoperatorio se recupera 1 ó 2 días antes en los pacientes que usan anestesia general más peridural $\mathrm{V} / \mathrm{s}$ anestesia general más morfina por IV-PCA $(P<0,005)^{24}$.

En relación a lo publicado, la función gastrointestinal generalmente se recupera a los 2-3 días antes en los pacientes con EAT + analgesia epidural postoperatoria.

\section{STRESS}

La respuesta de estrés frente a la cirugía, se inicia durante el preoperatorio, tiene su punto máximo en el postoperatorio y culmina al $3^{\text {er }} 0$ 4 o día postoperatorio. Esta respuesta, activa el sistema nervioso simpático y somático, produciendo una cascada de eventos fisiológicos y metabólicos predecible, lo que se manifiesta con taquicardia, hipertensión, aumento del consumo de oxígeno miocárdico, fiebre, inmnosupresión, metabolismo proteico aumentado e hiperglicemia25. El pick de respuesta al stress está fuertemente asociado a morbilidad postoperatoria cardíaca, vascular e infecciosa ${ }^{26,27}$

Yeager et al. ya en el año 1987 demostró que la anestesia peridural producía una reducción significativa en los niveles de cortisol urinario en las primeras $24 \mathrm{hrs}(\mathrm{P}=0,025)$ al compararla con otros tipos de anestesia6.

Actualmente nadie discute que la analgesia epidural torácica en cirugía abdominal reduce significativamente la magnitud de la respuesta al estrés al compararla con IV-PCA y AINE ${ }^{25,28}$.

\section{RESPUESTA INMUNE}

El deterioro de la respuesta inmune posquirúrgico está relacionado al aumento de las infecciones postoperatorias y a la progresión del cáncer ${ }^{3,28}$.
Son 3 los factores durante la cirugía que potencialmente pueden producir una falla de la respuesta inmune:

1. Los opioides endovenosos y la inhalación de anestésicos, disminuyen la actividad citotóxica de los Natural Killer (NK) 3,29.

2. La respuesta al stress quirúrgico, aumenta los glucocorticoides plasmáticos, suprime las células $T, B$, monocitos, neutrofilos y la activación de los NK 3,28,29.

La analgesia-anestesia epidural preserva la respuesta inmune postoperatoria al atenuar la respuesta al stress, reduciendo la concentración mínima alveolar de los anestésicos inalados y disminuyendo el uso de opioides endovenosos3.

\section{CONGNITIVO}

El efecto de la peridural sobre la función cognitiva de los pacientes operados es controversial. No hay estudios serios que lo demuestren. Se cree que al minimizar la cantidad de anestesia general, al permitir un mejor control del dolor con menos sedación y al aumentar la presión arterial de oxígeno durante el postoperatorio, contribuiría a mejorar el estado cognitivo de estos pacientes ${ }^{3}$.

\section{COMPLICACIONES ASOCIADAS AL USO DE LA AP}

Las potenciales complicaciones de la AAE son causa de la baja aceptación y el poco entusiasmo que se tiene por esta técnica. El daño neurovascular durante la colocación del catéter, las reacciones a la analgesia y a la anestesia local si bien son poco frecuentes, pueden ser permanentes. El riesgo máximo de daño neurológico permanente es de $0,07 \%$ (intervalo de confidencia de un $95 \%)^{30}$.

La punción accidental de la duramadre por el catéter o la inyección intratecal del anestésico local ocurre en un $0,6-1,3 \%^{30}$ y la subsecuente cefalea posdural en un $16-86 \%{ }^{3}$. Otros síntomas neurológicos transitorios como, dolor dorsal agudo o parestesia, pueden presentarse también ${ }^{4}$.

Los factores de riesgo para presentar síntomas neurológicos son: obesidad 1.6 (95\% $\mathrm{Cl}, 1$ to 2.5 ), uso de lidocaína (relative risk, 5.1; $95 \% \mathrm{Cl}, 2.5$ to 10.2 ), posición litotómica 2.6 (95\% $\mathrm{Cl}, 1.5$ to 4.5$)$ y pacientes ambulatorios 3.6 (95\% $\mathrm{Cl}, 1.9$ to 6.8 . $)^{31}$.

La meningitis y el absceso epidural (0:9232)1 son raros. Los factores de riesgo de meningitis 
son punción de duramadre, septicemia, catéter prolongado, técnica no estéril ${ }^{3}$.

La complicación más temida es la paraplejia. Esta se produce generalmente por un hematoma epidural por lesión de los vasos espinales durante la instalación o retiro del catéter en un paciente anticoagulado ${ }^{3}$. La incidencia de hematoma epidural es menor a $1 / 150.000^{1}$. Raramente es causado por un absceso espinal o el síndrome de arteria espinal anterior. El reconocimiento temprano y la laminectomía descompresiva dentro de las 8 hrs del diagnóstico disminuye las secuelas.

La neurotoxicidad del anestésico local se relaciona al tipo, concentración y absorción sistémica de él. Una alta absorción sistémica puede producir seizure, pérdida del reflejo protector de la vía aérea, depresión respiratoria, coma, arritmias cardíacas, inestabilidad hemodinámica con bloqueo motor y autonómico (retención urinaria) en un 0-45\% ${ }^{3}$. La hipotensión y bradicardia producida por la simpatolisis, se previene con la administración de fluidos preinducción, evitando el uso de lidocaína y el uso selectivo del fentanyl3.

La depresión respiratoria por los opioides ( $<1 \%$ de incidencia), se previene asociando al opioide un anestésico local, logrando disminuir la dosis del primero ${ }^{3}$.

Muchos critican la imposibilidad de dar profilaxis de TVP mientras se utiliza la epidural, sin embargo, hay estudios que han demostrado que es seguro hacerlo ${ }^{32}$.

\section{COSTO Y DURACIÓN DE HOSPITALIZACIÓN}

La epidural por sí sola no tiene efectos significativos sobre la duración de la hospitalización ${ }^{25}$; pero bajo un protocolo que incluya otras variables como alimentación precoz, movilización precoz, etc. que sólo se pueden lograr asociando el uso de esta técnica anestésica si se logra disminuir la estadía hospitalaria en al menos un día 33 .

\section{CONCLUSIÓN}

El uso de anestesia epidural intraoperatoria y postoperatoria está asociado a una disminución de la incidencia y severidad de las alteraciones fisiológicas perioperatorias y morbilidad postoperatoria. Esta técnica ha logrado demostrar que: acorta el íleo postoperatorio, disminuye las complicaciones respiratorias y cardiovasculares, disminuye la respuesta al stress, mejora la respuesta inmune y disminuye la incidencia de trombosis venosa.

Si bien las complicaciones de la AP, existen, éstas tienen muy baja incidencia, razón por la que los autores creemos que, de ningún punto de vista, los riesgos podrían ser mayores que los beneficios y, por lo tanto, este tipo de anestesia debiera ser utilizada en todos los pacientes que se les va a practicar una cirugía mayor abdominal, salvo en los que tengan alguna contraindicación formal de ésta.

\section{REFERENCIAS}

1. Wheatley RG, Schug SA, Watson D. Safety and efficacy of postoperative epidural analgesia BrJ Anaesth 2001; 87:47-61.

2. Hoffmann $V L$, Vercauteren $M P$, Vreugde J $P$, Hans GH, Coppejans HC, Adriaensen HA. Posterior epidural space depth: safety of the loss of resistance and hanging drop techniques. $\mathrm{Br} J$ Anaesth 1999; 83:807-9.

3. Moraca RJ , Sheldon DG, Thirlby RC. The role of epidural anesthesia and analgesia in surgical practice. Ann Surg 2003; 238:663-73.

4. Groeben $\mathrm{H}$. Epidural anesthesia and pulmonary function. J Anesth 2006; 20:290-299.

5. Blomberg S, Emanuelsson H, Kvist H, Lamm C, Pontén J , Waagstein F, et al. Effects of thoracic epidural anesthesia on coronary arteries and arterioles in patients with coronary artery disease. Anesthesiology 1990; 73:840-7.

6. Yeager MP, Glass DD, Neff RK, B rinck-J ohnsen T. Epidural anesthesia and analgesia in high-risk surgical patients. Anesthesiology. 1987; 66:729-36.

7. Beattie WS, Buckley DN, Forrest J B. Epidural morphine reduces the risk or postoperative myocardial ischaemia in patients with cardiac risk factors. Can J Anaesth 1993; 40:532-41.

8. Rodgers A, Walker N, Schug S, McKee A, Kehlet $H$, van Zundert $A$, et al. Reduction of postoperative mortality and morbidity with epidural or spinal anaesthesia: results from overview of randomised trials. BMJ 2000; 321:1493-6.

9. Ballantyne J C, Carr DB, deFerranti S, Suarez T, Lau J , Chalmers TC, et al. The comparative 
effects of postoperative analgesic therapies on pulmonary outcome: cumulative meta-analyses of randomized, controlled trials. Anesth Analg 1998; 86:598-612.

10. Park WY, Thompson J S, Lee KK, Department of Veterans Affairs Cooperative Study \#345 Study Group. Effect on epidural anesthesia and analgesia on perioperative outcome. A randomized, controlled Veterans Affairs cooperative study. Ann Surg. 2001; 234:560-9.

11. Meyers J, Lembeck L, O'Kane H, Baue AE. Changes in functional residual capacity of the lung after operation. Arch Surg 1975; 110:576-83.

12. Werawatganon T, Charuluxanun S. Patient controlled intravenous opioid analgesia versus continuous epidural analgesia for pain after intraabdominal surgery. Cochrane Database of Syst Rev 2005, CD004088.

13. Whaba WM, Don HF, Craig DB. Post-operative epidural analgesia: effects on lung volumes. Can Anaesth Soc J 1975; 22:519-27.

14. Mankikian B, Cantineau J P, Bertrand M, Kieffer $E$, Sartene $R$, Viars $P$. Improvement of diaphragmatic function by a thoracic extradural block after upper abdominal surgery. Anesthesiology 1988; 68:379-86.

15. Livingston EH, Passaro EP J r. Postoperative ileus. Dig Dis Sci 1990; 35:121-32.

16. Steinbrook RA. Epidural Anesthesia and Gastrointestinal Motility. Anesth Analg 1998; 86:83744.

17. Liu S, Carpenter RL, Neal J M. Epidural anesthesia and analgesia: Their role in postoperative outcome. Anesthesiology 1995; 82:1474-506.

18. Kapral S, Gollmann G, Bachmann D, Prohaska B, Likar R, J andrasist 0 , et al. The effects of thoracic epidural anesthesia on intraoperative visceral perfusion and metabolism. Anesth Analg 1999; 88:402-6.

19. Carpenter RL. Gastrointestinal benefits of regional anesthesia/analgesia. Reg Anesth 1996; 21:13-7.

20. Rimback G, Cassuto J , Tollesson PO. Treatment of postoperative paralytic ileus by intravenous lidocaine infusion. Anesth Analg 1990; 70: 414-9.

21. Thörn SE, Wickbom G, Philipson L, Leissner $P$, Wattwil $M$, et al. Myoelectric activity in the stomach and duodenum after epidural administration of morphine or bupivacaine. Acta Anaesthesiol Scand 1996; 40:773-8.

22. Liu SS, Carpenter RL, Mackey DC, Thirlby RC, Rupp SM, Feinglass NG, et al. Effects of perioperative analgesic technique on rateo of recovery after colon surgery. Anesthesiology 1995; 83(4): 757-65.

23. Seeling W, Bruckmooser K, Hufner C, Kneitinger $\mathrm{E}$, Rigg C, Rockemann $\mathrm{M}$. Continuous thoracic epidural analgesia does not diminish postoperative complications after abdominal surgery in patients at risk. Anaesthesist 1990; 39:33-40.

24. Taqi A, Hong X, Mistraletti G, Stein B, Charlebois $P, C$ arli $F$. Thoracic epidural analgesia facilitates the restoration of bowel function and dietary intake in patients undergoing laparoscopic colon resection using a traditional, nonaccelerated, perioperative care program. Surg Endosc 2007; 21:247-52.

25. Kehlet H, Holte K. Effect of postoperative analgesia on the surgical outcome. Br J Anaesth 2001; 87:62-72.

26. Carli F, Webster J, Pearson M, Pearson J, Bartlett $S$, B annister $P$, et al. P rotein metabolism after abdominal surgery: effects of 24-h extradural block with local anesthetic. Br J Anaesth 1991; 67:729-34.

27. Naito Y, Tamai S, Shingu K, Shindo K, Matsui $T$, Segawa $H$, et al. Responses of plasma adrenocorticotropic hormone, cortisol, and cytokines during and after upper abdominal surgery. Anesthesiology 1992; 77:426-31.

28. Kawasaki T, Ogata M, Kawasaki C, Okamoto $K$, Sata T. Effects of epidural anaesthesia on surgical stress-induced immunosuppression during upper abdominal surgery. Br J Anaesth 2007; 98:196-203.

29. Yokoyama M, Itano Y, Mizobuchi S, Nakatsuka $H$, Kaku R, Takashima $T$, et al. The effects of epidural block on the distribution of lymphocyte subsets and natural-killer cell activity in patients with and without pain. Anesth Analg 2001; 92: 463-9.

30. Giebler RM, Scherer RU, Peters J . Incidence of neurologic complications related to thoracic epidural catheterization. Anesthesiology 1997; 86: 55-63.

31. Freedman J M, Li D, Drasner K, J askala M, Larsen B, Wi S. Transient neurologic symptoms after spinal anesthesia: an epidemiologic study of 1863 patients. Anesthesiology 1998; 89:633-41.

32. Schwander D, Bachmann F. Heparin and spinal or epidural anesthesia: decision analysis. Ann $\mathrm{Fr}$ Anesth Reanim 1991; 10:284-96.

33. Bradshaw BG, Lui SS, Thirlby RC. Standardized perioperative care protocols and reduced length of stay after colon surgery. J Am Coll Surg 1998; 186:501-6. 